\title{
Assessment of Epstein-Barr virus nucleic acids in gastric but not in breast cancer by next-generation sequencing of pooled Mexican samples
}

\author{
Ezequiel M Fuentes-Pananá1, Violeta Larios-Serrato², Alfonso Méndez-Tenorio², \\ Abigail Morales-Sánchez ${ }^{1}$, Carlos F Arias' 3 , Javier Torres ${ }^{4 /+}$ \\ ${ }^{1}$ Hospital Infantil de México Federico Gómez, Unidad de Investigación en Virología y Cáncer, México, DF, México \\ ${ }^{2}$ Escuela Nacional de Ciencias Biológicas, Unidad Profesional Lázaro Cárdenas, Laboratorio de Biotecnología y Bioinformática Genómica, \\ México, DF, México ${ }^{3}$ Universidad Nacional Autónoma de Mexico, Instituto de Biotecnología, \\ Departamento de Genética del Desarrollo y Fisiología Molecular, Cuernavaca, Morelos, México ${ }^{4}$ Centro Médico Nacional Siglo XXI, \\ Hospital de Pediatría, Unidad de Investigación Médica en Enfermedades Infecciosas y Parasitarias, México, DF, México
}

Gastric $(G C)$ and breast $(B r C)$ cancer are two of the most common and deadly tumours. Different lines of evidence suggest a possible causative role of viral infections for both GC and BrC. Wide genome sequencing (WGS) technologies allow searching for viral agents in tissues of patients with cancer. These technologies have already contributed to establish virus-cancer associations as well as to discovery new tumour viruses. The objective of this study was to document possible associations of viral infection with GC and BrC in Mexican patients. In order to gain idea about cost effective conditions of experimental sequencing, we first carried out an in silico simulation of WGS. The next-generation-platform IlluminaGallx was then used to sequence GC and BrC tumour samples. While we did not find viral sequences in tissues from BrC patients, multiple reads matching Epstein-Barr virus (EBV) sequences were found in GC tissues. An end-point polymerase chain reaction confirmed an enrichment of EBV sequences in one of the GC samples sequenced, validating the next-generation sequencing-bioinformatics pipeline.

Key words: gastric cancer - breast cancer - gastritis - wide genome sequencing - EBV

Globalisation, climate change, urbanisation of wild areas, and other modern conditions of life are contributing to an increased exposure to a number of infectious agents. Concomitantly, worldwide populations are experiencing a demographic transition that has placed cancer as one of the leading causes of death. To date, a number of pathogens, mostly viruses, have been classified as carcinogenic to humans by the International Agency for Research on Cancer. These viruses include: high-risk human papillomavirus (HPV), Epstein-Barr virus (EBV), Kaposi sarcoma herpesvirus, Merkel cell polyomavirus (MCPyV), hepatitis $\mathrm{B}$ virus, hepatitis $\mathrm{C}$ virus, and human T-lymphotropic virus 1 (Morales-Sánchez \& Fuentes-Pananá 2014). Most families of oncogenic viruses are either strict DNA or viruses in which their life cycle has a DNA stage, such as retroviruses, which convert their genomic RNA into proviral DNA that is integrated into the host genome.

It is estimated that human cancers of viral aetiology comprise up to $20 \%$ of all tumours, with higher frequencies found in developing countries (Crawford 2005). However, viral agents often defy isolation and recognition by

doi: 10.1590/0074-02760150405

Financial support: CONACyT (69450 - to J Torres), Fondo SEP CONACyT (176880 - to EMF-P), FIS/IMSS/PROT/G12/1120 (to EMF-P), Fondo de Apoyo a la Investigación HIM (2013-051 - to EMF-P) EMF-P and VL-S contributed equally to this work.

+ Corresponding author: jtorres157@yahoo.com.mx

Received 22 October 2015

Accepted 12 January 2016 traditional culture or molecular methods. Next-generation sequencing (NGS) technologies are currently used for wide-genome sequencing (WGS) providing high throughput data from single genomes, making them optimal to interrogate for the presence of known and previously unrecognised viral agents. Furthermore, WGS has recently allowed the identification of a novel polyomavirus associated with Merkel cell carcinoma (MCC) (Feng et al. 2008).

Gastric (GC) and breast (BrC) cancer are two of the most common cancers and two of the most important problems in public health now days. GC is the fifth most frequent cancer worldwide and the third cause of death by cancer, whilst $\mathrm{BrC}$ is the most frequent cancer in working-age woman (Ferlay et al. 2014). GC is considered primarily of infectious aetiology, with Helicobacter pylori infection recognised as the most important risk factor (Crew \& Neugut 2006). Colonisation of the gastric mucosa by $H$. pylori triggers a chronic inflammatory response that when unregulated chronically damages the gastric mucosa, generating progressive lesions of increased severity and risk of ending in cancer (Correa et al. 2006). Lesions usually start with a nonatrophic gastritis (NAG), progress to atrophic gastritis, intestinal metaplasia and dysplasia, to finally evolve into GC (Correa et al. 2006). More recently, several lines of evidence also support infection by EBV as an important causative agent for GC (Murphy et al. 2009, Camargo et al. 2011).

Several risk factors have also been described for $\mathrm{BrC}$, including a family and personal history of $\mathrm{BrC}$, increased number of menstrual cycles, reproductive history, hormone therapy, cigarette smoking, and obesity (Kubista 2001). Viral infection has also been suggested as a risk factor for $\mathrm{BrC}$, and mouse mammary tumour vi- 
rus (MMTV), HPV and EBV are the agents reported as probably associated with $\mathrm{BrC}$ (Joshi \& Buehring 2012). However, data have been highly variable, with reported infection prevalence ranging from $0-100 \%$ and the viral infection- $\mathrm{BrC}$ association remains highly controversial.

In this study, we searched for fingerprints of viral infection in pools of $\mathrm{GC}$ and $\mathrm{BrC}$ tissues using the IlluminaGallx NGS platform. Previously, we implemented an in silico NGS simulation assay aimed to find a manageable cost effective pipeline of analysis to interrogate for the presence of viral sequences in cancer samples. We did not find sequences supporting viral participation in breast tumours, whereas multiple reads matching EBV sequences were found in gastric tumours. An endpoint polymerase chain reaction (PCR) confirmed EBV sequences in one of the GC samples sequenced ratifying the utility of the bioinformatics pipeline of analysis. The development and implementation of specific and sensitive NGS together with bioinformatics strategies will become critical to dissect the biome associated with cancer and many other diseases of infectious origin.

\section{SUBJECTS, MATERIALS AND METHODS}

Study population - Patients with confirmed diagnosis of $\mathrm{GC}, \mathrm{BrC}$, and NAG were included in the study. Five patients formed every study group. All patients were recruited in Mexico City, GC and $\mathrm{BrC}$ patients from the Oncology Hospital and patients with a NAG diagnosis from the Specialities Hospital, both from Mexican Institute of Social Security (IMSS) at the XXI Century National Medical Center. Tumour and tumour-adjacent tissues were derived from the organ resection. Tumour-adjacent tissues served as controls for specificity of tumour cell infection and these control samples were taken $\geq 2 \mathrm{~cm}$ apart from the tumour mass from the same patient in which tumour tissue was obtained. Gastric biopsies were from patients referred to the gastroenterology unit of the Specialities Hospital because of gastric symptoms. A fragment of all tissues was fixed in formaldehyde and embedded in paraffin and a slide was stained with haematoxylin-eosin and analysed by a pathologist to confirm the diagnosis. All tumour tissues included in the study were carcinomas with at least $70 \%$ of tumour cells; all GCs included in the study were classified as mixed type (intestinal and diffuse) according to the Lauren's criteria. All BrC were classified as ductal infiltrating. The $\mathrm{BrC}$ molecular classification was the following: three patients were luminal $\mathrm{A}$, one patient was with human epidermal growth factor receptor 2 positive, and one patient was triple negative. In the gastritis samples, we included cases without atrophy or pre-neoplastic lesions with a diagnosis of NAG.

Sample preparation - Ten milligrams of each tissue sample were disrupted in a TissueLyser II (Qiagen, Germany) for $20 \mathrm{~s}$ and homogenates were subjected to DNA purification with QIAamp DNA mini kit in a QIAcube automated sample processing workstation (Qiagen). Purified DNA was quantified using a spectrophotometer NanoDrop 1000 (Thermo Fisher Scientific, USA) and DNA quality was determined with the 260/280 ratio of absorb- ance, integrity by electrophoresis in agarose gels, and by PCR of $\beta$-actin (670 bp) endogenous gene using primers previously described (Fuentes-Pananá et al. 2004).

Sample analysis - DNA from $\mathrm{BrC}, \mathrm{BrC}$ tumour-adjacent controls, GC, GC tumour-adjacent controls, and NAG was sequenced. Pools of five patients formed every group of study for a total of five pools. We used $1 \mu \mathrm{g}$ of DNA from each patient for sequencing a total of $5 \mu \mathrm{g}$ per group. DNA from each group was loaded into separated lanes of a flow cell from a Genome Analyzer IIx (Illumina, USA). Sequencing was performed through 36 cycles of single base pair extensions. Fluorescent images were analysed using the Illumina base calling pipeline v.1.4 to obtain data sequences. The resulting initial sequences of the samples have a length of 36-mer. Those reads obtained for all samples (in FASTQ format) were filtered from undesired sequences using the assembly Perl tools from the Euler-SR program (Chaisson \& Pevzner 2008). Then, a collection of programs developed with the Lazarus Free Pascal programming language was used to (i) eliminate sequences of low complexity such as mononucleotide repeats, (ii) trim end-nucleotides that did not fulfill a phred quality value $<30$, (iii) change the data file format from FASTQ to FASTA, and (iv) eliminate repeated reads.

In silico preliminary analysis - Simulation of Illumina Sequencing was carried out in ART (Huang et al. 2012) with the ART's parameterised quality profiles and model error specific of the platform. The inputs contained two copies of human genome (GRCh37.p13) plus one of the following options: (i) 100 copies of the HPV 16 genome (NC 001526.2) (Theelen et al. 2010), (ii) 10 copies of the MMTV genome (NC_001503.1) (Morris et al. 1977), or seven copies of the EBV genome (NC 009334.1) (Liu et al. 2011). Emulation generated synthetic Illumina sequencing reads according to different covertures $(0.1 \mathrm{X}, 0.2 \mathrm{X}, 0.5 \mathrm{X}$ and $1 \mathrm{X})$ and two different read sizes (36 mer and 100 mer). Subtraction of poor quality and human sequences, as well as mapping of viral sequences, is described in the next section.

Pipeline bioinformatics analysis - This pipeline of analysis was carried out for the in silico data first and served as a guidance to set the conditions of the sample sequencing and analysis. Here, the search for reads matching viral genomic sequences was performed according to the method developed by Aleksandar (Kostic et al. 2011). Reads were compared against human sequences using the Bowtie short read aligner v.0.12.7 (Langmead et al. 2009) considering 2, 1, or 0 mismatches, and after a preliminary analyses only 1 mismatch was allowed throughout the final analysis. A human genome databank was created for this comparison, which contained five different genomic databases: three derived from male assemblies hs_ref_GRCh37.p2 (2009), hs_refHuRef (2007), and hs_alt_Celera (2001) (ftp.ncbi. nih.gov/genomes/H_sapiens/), one female assembly (2008) (ftp.1000genomes.ebi.ac.uk), one mitochondrial (2010) (NC 012920.1), plus two transcriptome databases: National Center for Biotechnology Information (NCBI) Homo sapiens RNA database (ftp.ncbi.nih.gov/ 
genomes/H_sapiens/RNA/) and Ensembl Homo sapiens cDNA database (ftp.ensembl.org). The subtraction of human reads was done with a suite of PERL developed programs. The remaining non-human reads were analysed by BLASTN (v.2.2.28, word size $=9, \mathrm{E}$-value $\left.=1 \times 10^{-6}\right)$ to $1,520,849$ viral sequences [downloaded from NCBI Nucleotide (ncbi.nlm.nih.gov/nucleotide)] using the search term "viruses" [porgn: txid10239] on 1 January 2013. The viral reads obtained were contrasted against a dataset of bacterial, protozoa, and fungi sequences to confirm their authenticity using Bowtie (parameter 1 mismatch). The dataset was a compilation of the following: 3,336 bacteria complete genomes (2012) (ftp://ftp. ncbi.nih.gov/genomes/Bacteria/), 500 human microbiome bacteria (ftp.ncbi.nih.gov/genomes/HUMAN_MICROBIOM/Bacteria/), 716,562 protozoa sequences (NCBI Nucleotide database terms: "apicomplexans" [porgn: txid5794], "amoebozoa" [porgn: xid554915], diplomonadida [porgn: txid5738], "kinetoplastida" [porgn: txid5653], "platyhelminthes" [porgn:_txid6157]), and 522,571 fungi sequences (NCBI nucleotide database terms: "ascomycetes" [porgn: txid4890], "neocallimastigales" [porgn: txid29006], "microsporidians" [porgn: txid6029], "mucorales" [porgn: txid4827], "glomerales" [porgn: txid1028384], "tremellomycetes" [porgn: txid155616]). All generated sequences were deposited in SRA database with the next BioSample accessions 774161 (BrC), 795877 (BrC control), 795890 (GC), 796157 (GC control), and 796243 (gastritis). The analysis pipeline was constructed using the Perl 5.8.1 program. Data processing was carried out in a Mac Pro equipment with a Mac OS X server operating system, a $2 \times 2.66 \mathrm{GHz}$ 6-core Intel Xeon processor, and 32 GB RAM memory.

Direct search of viral sequences - To corroborate the lack of hits for viral agents previously documented in samples, 6,782 sequences of HPV [porgn: txid10566], 258 sequences of mouse mammary tumour virus [porgn: txid11901], and 814 sequences of bovine leukaemia virus [porgn: txid11901] were downloaded and matches were directly searched in the $\mathrm{BrC}$ and breast nontumour control databases. Sequences were aligned using Bowtie, allowing up to 1 mismatch and without removing any human sequence; the only filters used in this latter search were quality phred, mononucleotides, and repeated sequences of data sets.

$P C R$ detection of EBV - DNA samples were subjected to a first PCR with primers LLW1 and LLW2 (Labrecque et al. 1995) which amplify a region within the BamHI W fragment in the EBV genome. The Daudi cell line was used as positive control. The PCR mix $(50 \mu \mathrm{L})$ contained $200 \mathrm{ng}$ of template DNA, $200 \mu \mathrm{M}$ of dNTPs mix, $2.5 \mathrm{mM}$ of $\mathrm{MgCl}_{2}, 5 \mu \mathrm{L}$ of Taq Polymerase buffer 10x with $\left(\mathrm{NH}_{4}\right)_{2} \mathrm{SO}_{4}, 200 \mathrm{nM}$ of each primer, and $2.5 \mathrm{U}$ of Taq Polymerase (all from Thermo Fisher Scientific). The PCR reaction was: an initial denaturation step of $5 \mathrm{~min}$ at $94^{\circ} \mathrm{C}$ and then 30 cycles of $94^{\circ} \mathrm{C}$ for $1.5 \mathrm{~min}$, $57^{\circ} \mathrm{C}$ for $45 \mathrm{~s}$ and $72^{\circ} \mathrm{C}$ for $1 \mathrm{~min}$, and a final extension of $72^{\circ} \mathrm{C}$ for $7 \mathrm{~min}$. Internal primers to the first PCR amplicon were designed for a nested PCR: LLWint1 5'CTTTGTCCAGATGTCAGGGG3' and LLWint2 5'GCCT-
GAGCCTCTACTTTTGG3'. The $50 \mu \mathrm{L}$ PCR mixture contained $1 \mu \mathrm{L}$ of the first PCR (1:1000 final dilution), $200 \mu \mathrm{M}$ of dNTPs mix, $2.5 \mathrm{mM}$ of $\mathrm{MgCl}_{2}, 5 \mu \mathrm{L}$ of Taq Polymerase buffer $10 \mathrm{x}$ with $\left(\mathrm{NH}_{4}\right)_{2} \mathrm{SO}_{4}, 400 \mathrm{nM}$ of each primer, and $2.5 \mathrm{U}$ of Taq Polymerase (all from Thermo Fisher Scientific). The reaction was performed with an initial denaturation step at $94^{\circ} \mathrm{C}$ for $5 \mathrm{~min}$, followed by 15 cycles of $94^{\circ} \mathrm{C}$ for $20 \mathrm{~s}, 57^{\circ} \mathrm{C}$ for $20 \mathrm{~s}$, and $72^{\circ} \mathrm{C}$ for 30 $\mathrm{s}$, and a final extension of $72^{\circ} \mathrm{C}$ for $7 \mathrm{~min}$.

Sanger sequencing - The identity of the EBV positive PCR product was confirmed by sequencing both forward and reverse strands. The PCR product was purified using QIAquick gel extraction kit (Qiagen) according to manufacturer's instructions, and sequencing of the isolated DNA fragment was carried out in the Biology Institute, National Autonomous University of Mexico. Sequences were compared with the GenBank database using the BLAST program (Altschul et al. 1990).

Ethics - The National Commission of Scientific Research and the Ethical Committee on Research of the IMSS approved this project. All patients were informed on the nature of the study and those willing to participate signed a written informed consent prior to specimen collection.

\section{RESULTS}

Preliminary simulation analysis - Looking to implement a bioinformatics pipeline that allowed manageable and cost effective sequencing conditions, we first carried out an NGS simulation analysis. We selected three previously $\mathrm{GC}$ and $\mathrm{BrC}$-associated viruses with different genome sizes, ranging from about 8,800 bp (MMTV) to $172,000 \mathrm{bp}$ (EBV). Three different inputs containing human and viral genomes were constructed. The number of human and viral genomes used in every input was adjusted mimicking real infected tumour cells. Thus, 100 copies of HPV 16 genome (Theelen et al. 2010), 10 copies of MMTV genome (Morris et al. 1977), or seven copies of EBV genome (Liu et al. 2011) were used per diploid human genome. Generation of synthetic reads was followed by several rounds of filtration (See Fig. 1 and Subjects, Materials and Methods). Multiple viral reads mapped to filtered data sets. This analysis predicted the minimal coverage to detect viral fingerprints. We also interrogated in silico for the most suitable size read, and a 36 mer vs. a 100 mer read size was compared (not shown). This simulation showed that using 36 mer read size was enough to detect viral hits (Fig. 1). This preliminary analysis guided us about adequate conditions of sequencing for viral detection in biological samples.

$N G S$ in biological samples - Looking to reduce analysis cost, pools of samples were sequenced based on conditions founded in the preliminary analysis. Our models of study were $\mathrm{GC}$ and $\mathrm{BrC}$ since multiple lines of evidence support an infectious aetiology for both types of cancer. Also, since most families of tumour viruses can be traced by DNA, DNA samples were sequenced. Looking to strengthen the capacity to find possible viral sequences, only tumour tissues containing at least $70 \%$ of tumour cells were included. Tumour-adjacent tissues 


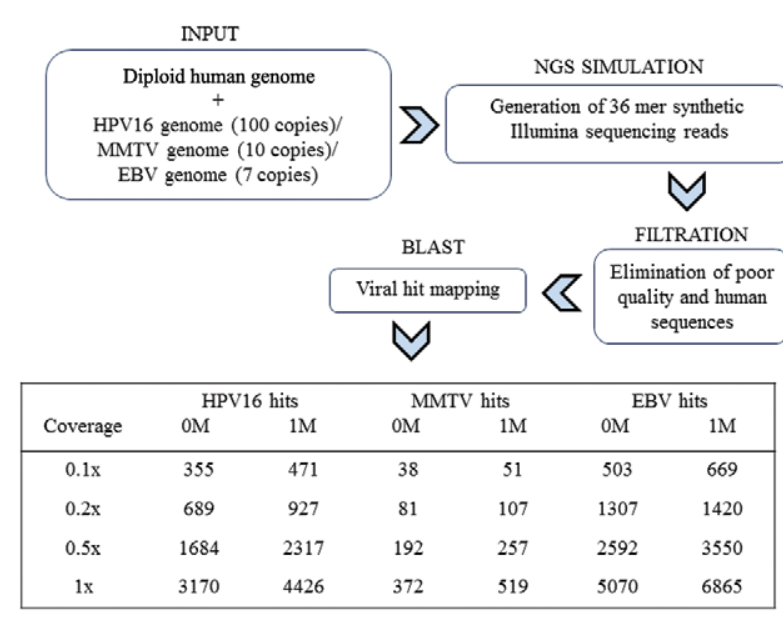

Fig. 1: flowchart of next-generation sequencing (NGS) simulation. Illumina sequencing emulation generating 36 or 100 mer (not shown) reads was run in ART (Huang et al. 2012). Different coverages were tested. Several viral hits from human papillomavirus (HPV) 16, mouse mammary tumour virus (MMTV), and Epstein-Barr virus (EBV) were mapped from human-viral inputs mimicking genomic load present in tumours associated to these agents. Viral sequences were screened allowing $0(0 \mathrm{M})$ or $1(1 \mathrm{M})$ mismatch. Tens or hundreds of viral reads were found.

isolated from the same cancer patients and located at least $2 \mathrm{~cm}$ apart from the tumour mass served as controls for specificity of tumour cell infection, while biopsies of patients with NAG addressed the possible viral participation in early inflammatory precursor lesions.

The pipeline analysis of the data generated by the Illumina GAIIx sequencing and the percentage of non-human reads remaining after each level of filtration are shown in Fig. 2. Ten to $20 \%$ of sequences were eliminated on the bases of quality and composition, $76-88 \%$ reads preferentially matched human sequences and were also eliminated, to end with an average of $2.39 \%$ non-human reads for all tissues sequenced. Table I shows the number of initial and final non-human reads after all filtration steps. Human sequences were a compendium of seven different databases, which allowed a more stringent tool for filtration and a better selection of sequences with more distant similarities to human genomes. The database of human sequences can be found freely at NCBI and European Molecular Biology Laboratory, and all human sequences found in pools of sequenced tissues at drive.google.com/folderview?id=0B3AZ9N8M5ZMfVGtpbUFtQ1JBbE0\&usp=sharing for readers use.

The number of non-human reads obtained constituted a set of manageable information for more robust analysis; such reads were blasted against different microbial databases, including a database of 1,520,849 viral sequences. The viral database was based in available sequences deposited in the NCBI, and it is also freely available (drive.google.com/folderview? id=0B3AZ9N8M5ZMfYjJCT0xlNzZSdkU\&usp=sharing).

Different types of viral hits were found, most of them were of no interest since they were not from members of a family of tumour viruses or because of they were present across all tumour and control tissues. For instance, several hits matched sequences present in phages or vectors that are commonly used as tools for molecular biology research: eight-18 hits matching enterobacteria phage sequences were found across all sequenced tissues, one-two hits matching baculovirus sequences were also found across all tissues, and one hit matching SAdV-40 was found in $\mathrm{BrC}$ tissue. The origin of this genetic material most likely comes from contamination with enzymes and reagents used to process tissues. Two hits were found matching the Torque teno virus, which is commonly found in cells of the immune system: one in $\mathrm{BrC}$ tumour-adjacent control and one in GC. Slightly more interesting was to find hits matching retroviral sequences; although, those hits showed higher similarity with human endogenous retrovirus $\mathrm{H}$ and $\mathrm{K}$ and less to oncogenic MMTV, which has been linked to $\mathrm{BrC}$. Furthermore, these retroviral hits were found in all tissues sequenced as shown in Table II.

Several hits matching members of the Herpesviridae family were found: six against human herpesvirus type 7 (HHV7), one in BrC tumour-adjacent, one in GC, and four in NAG; two against citomegalovirus, one in $\mathrm{GC}$, and one in GC tumour-adjacent; six against herpes simplex virus type 1 (HSV1), one in $\mathrm{BrC}$, one in $\mathrm{GC}$ tumour-adjacent, and four in GC. These HSV1 sequences also exhibited high similarity ( $>90 \%)$ to other members of the Alphaherpesvirinae subfamily, to gallid herpesvirus 2 (infects birds), angullid herpesvirus (infects eels), and cyprinid and Koi herpesvirus (both infect fish).

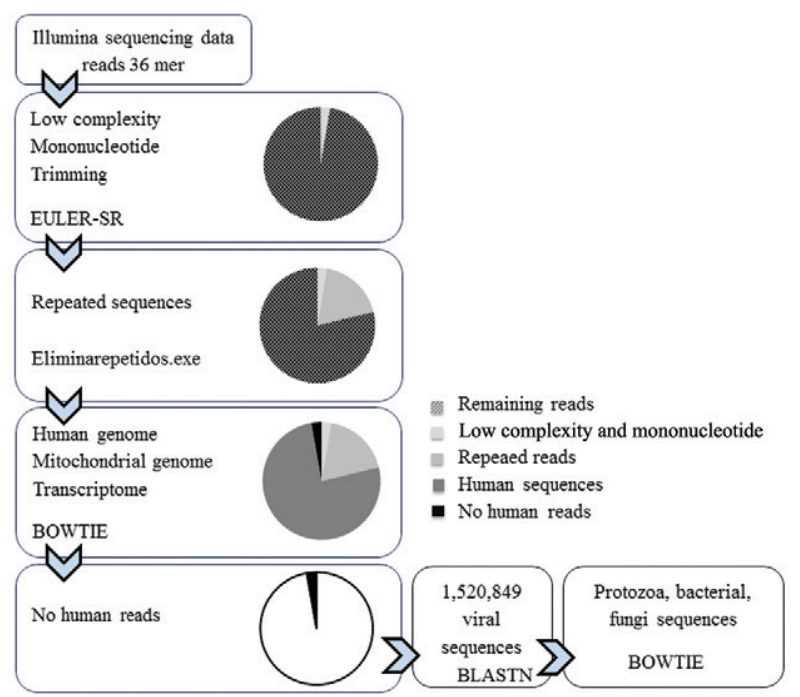

Fig. 2: pipeline of analysis of deep sequencing data. next-generation sequencing crude data were enriched of viral sequences by subtracting unwanted reads as follows: (i) filtering low-quality reads by EULER-SR, (ii) repeated sequences by Eliminarepetidos.exe, (iii) human sequences aligning by BOWTIE, (iv) other nonviral reads by aligning with BLASTN against publicly available sequence databases (protozoan, bacterial, and fungi sequences). Pie charts illustrate the subtracted and remaining non-human reads. For a more in deep explanation see materials and methods. 
Hits matching HHV type 6 (HHV6) and EBV sequences exhibited tissue specificity; HHV6 had 61 hits in breast and zero in gastric tissue. However, there was

TABLE I

Number of reads

\begin{tabular}{lcc}
\hline Tissue & Total reads & Non-human reads \\
\hline BrC & $15,600,870$ & 269,740 \\
BrC control & $30,100,894$ & 513,073 \\
GC & $29,213,391$ & 770,760 \\
GC control & $27,296,544$ & 507,667 \\
Gastritis & $14,421,599$ & 329,850 \\
\hline
\end{tabular}

$\mathrm{BrC}$ : breast cancer; GC: gastric cancer. not specificity of infection for the tumour sample (16 hits in $\mathrm{BrC}$ and 45 in $\mathrm{BrC}$ tumour-adjacent control tissue); therefore, this result does not support a direct etiological role for HHV6 in BrC. On the other hand, EBV had 10 hits in GC, while only one in GC tumour-adjacent tissue and two in NAG. Table III shows the identity of the gene targets of the EBV hits. One hit overlaps to two overlapping coding sequences: EBNA-2 and BYRF1.

No evidence of viral participation in $\mathrm{BrC}$ was found even though several lines of evidence point out for a possible participation of HPV, MMTV, bovine leukaemia virus, and EBV in the aetiology of this cancer. To further support our previous observation, direct searches of these viruses against the tumour and control tissues sequences were performed with similar results; no hits for these viruses were found. In contrast, when EBV sequences were contrasted against unfiltered sequences from all tissues, 103 hits were found in GC, 12 hits in adjacent control, and 29 hits in gastritis samples.

TABLE II

Viral hits

\begin{tabular}{lccccc}
\hline Virus & BrC & BrC control & GC & GC control & Gastritis \\
\hline EBV (HHV4) & 0 & 0 & 10 & 1 & 2 \\
HHV6 & 16 & 45 & 0 & 0 & 0 \\
HHV7 & 0 & 1 & 1 & 0 & 4 \\
CMV (HHV5) & 0 & 0 & 1 & 1 & 0 \\
Other herpesviruses & 1 & 6 & 7 & 7 & 0 \\
HERVS & 10 & 0 & 5 & 5
\end{tabular}

BrC: breast cancer; CMV: citomegalovirus; EBV: Epstein-Barr virus; GC: gastric cancer; HERVS: human endogenous retrovirus; HHV: human herpesvirus.

TABLE III

Identity of Epstein-Barr virus hits

\begin{tabular}{lcc}
\hline Tissue & Read & Target gene \\
\hline GC & TCACGGCATCTGGGGTGACCGGGGCCATCGGGTTT & BFLF2 \\
GC & GATGCCCTCCAGGTCAAAGACGTTGGAGGACGCT & BALF4 \\
GC & AGGGAGTCACGTAGGCACTAGACTCTTCATGTGAG & BART miRNA \\
GC & GGTTGAGGTGGTAAAGACGTGGGCCGTGGTCAGAT & BGLF2 \\
GC & GTTACATGGGGGACAAACATATCATCTAATTGTG & EBNA-2/BYRF1 \\
GC & ACCGGCGTGCGAGGAGCAGCATGCAGGCTCGGGCG & BPLF1 \\
GC & GGGGGCGGCCGGCCAGGAAGCTCTTGACCAGGTAG & BPLF1 \\
GC & CTGACTTTCATGAAATCCCTGACCTCGATGACTCC & BRRF2 \\
GC & CCCTACTTGGGAGAGTCCGGCAAGGCCAGAGACAC & BMRF2 \\
GC & TCCGGGGTAAGCTTCGGCCATGGCCGGAGCTCGTC & LF1 \\
GC control & CTTTGCGGCCCCGACTATCGACCCTGCATTTGCGA & BPLF1 \\
Gastritis & TCCTCGGAGCCAGCCCCCGGGGTTGGTTCTGCCCC & EBNA-LP \\
Gastritis & AAGATGCGAGTTTGCAATGTCCCCCGCCTCATCAA & BCRF1
\end{tabular}

GC: gastric cancer. 
Although, the main goal of this study was to analyse the virome of $\mathrm{BrC}$ and $\mathrm{GC}$, but since H. pylori infection is recognised as the main risk factor to develop NAG and GC, a blast of the non-human reads found in gastric lesions was performed against a database of 3,336 full bacterial genomes. These data is shown in Supplementary Figure. Hits mapping to the Helicobacter genus were found in $2 \%, 12 \%$, and $36 \%$ of all bacterial reads in GC, GC adjacent tissue control and NAG samples, respectively. Helicobacter was the most abundant genera found in gastritis, while Paracoccus and Propionobacterium were the most represented in GC and GC controls. These data is congruent with the literature documenting that $H$. pylori tents to be absent of tumour tissues, while it is highly abundant in early gastric lesion (Kokkola et al. 2003, Camorlinga-Ponce et al. 2008).

$E B V$ detection by $P C R$ - To confirm the presence of EBV in GC tumours and validate the results from the WGS, a PCR test was implemented using primers LLW1 and LLW2 (Labrecque et al. 1995) in a reaction set to detect $\geq 40,000$ viral genomes (Martínez-López et al. 2014). Tissues from the five GC tumours and their counterpart tumour-adjacent controls and the five NAG samples were individually tested by the PCR. Furthermore, since EBV usually resides in B cells in a low number of infected cells, mononuclear cells isolated from peripheral blood of GC and NAG patients were also included in the analysis. Fig. 3 shows the result of the PCR test; one of the GC tissues was found positive for EBV sequences, while the other four patients were negative. The patient positive by the PCR test was negative in the tumour-adjacent control tissue and peripheral mononuclear cells, supporting an

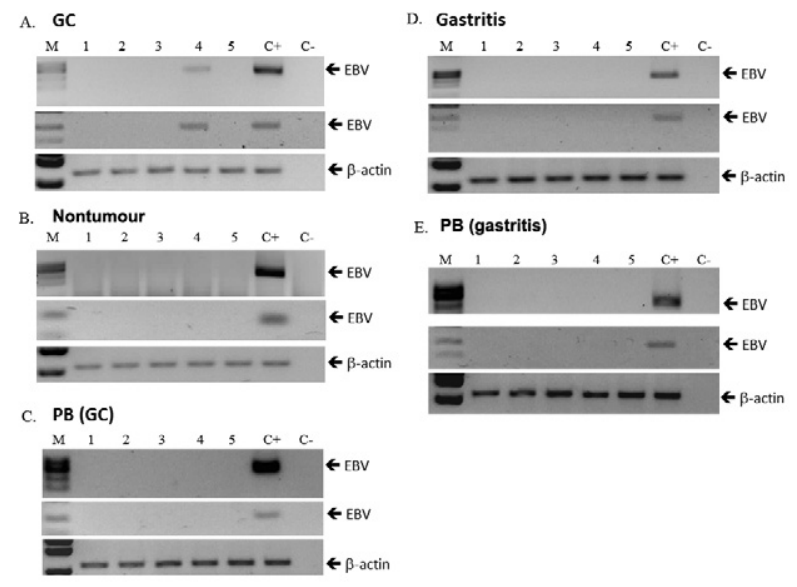

Fig. 3: detection of Epstein-Barr virus (EBV) by polymerase chain reaction (PCR). A: one of five gastric cancer (GC) samples was positive (sample four) to EBV; by first round PCR (upper panel) and nested PCR (middle panel); B: nontumour control tissues (C) and peripheral blood (PB) from the same GC patients were negative to EBV by both PCRs; $\mathrm{D}$ : none of the gastritis samples (E) nor PB of these patients were positive to EBV by any PCR. DNA from Daudi cells was used as positive control (C+). A reaction without DNA was used as negative control (C-); M: molecular weight marker. Lower panels shows the PCR of loading and DNA integrity control, the endogenous gene $\beta$-actin. enrichment of EBV infected cells in the tumour tissue. None of the NAG patients were positive, in agreement with the NGS data. EBV sequences were confirmed with a more sensitive nested PCR (detects $\geq 1,500$ viral particles) (Martínez-López et al. 2014) and by Sanger sequencing of the first positive PCR-amplicon.

Taken together, these data highlight the importance of NGS technologies as a powerful and manageable tool to interrogate cancer tissues for the presence of viral sequences looking to better understand the aetiology of the disease.

\section{DISCUSSION}

NGS technologies have opened new perspectives for viral research and diagnostic in multiple human and veterinary diseases. In recent years, this technology has allowed the identification and characterisation of new viruses, such as the Bundiubugyo virus, a virus related to Ebola and responsible for severe haemorrhagic fevers in humans (Towner et al. 2008), and an arenovirus closely related to lymphocytic choriomeningitis viruses (Kim et al. 2011), associated with fatal post-transplant disease. MCC was a long suspected cancer of infectious aetiology because it develops preferentially in immunosuppressed individuals. However, the identity of the causative agent remained elusive until high throughput sequencing and transcriptome subtraction methodologies allowed the identification of a novel polyomavirus in MCC samples (Feng et al. 2008). Now, it has been firmly documented that $\mathrm{MCPyV}$ is responsible for up to $100 \%$ of MCC (Agelli et al. 2010).

Here, we used a similar approach to interrogate for the presence of viral sequences in $\mathrm{BrC}$ and $\mathrm{GC}$ tissues, two of the most common cancers. However, considering that NGS-based methodologies result in an enormous set of data difficult for processing and interpretation, we implemented some strategies to reduce both cost and time of analysis to the experimental and bioinformatics approach. DNA samples were pooled (with DNA from 5 patients in every lane of the sequencer), thus, we reduced the cost of massive sequencing. Additionally, we carried out an in silico preliminary analysis simulating several NGS conditions according to the reported viral genome copies found in cancer. Through this analysis, we selected an affordable but sufficient coverage to detect viral sequences.

A database of multiple human sequences was constructed and used to digitally subtract candidate sequences of viral origin, especially those matching members of viral families with oncogenic characteristics. This strategy leaded us to find HHV6 sequences associated with breast tissue and EBV with gastric tumour samples. GC has been extensively associated with $H$. pylori infection and more recently with EBV, and we found evidence of both pathogens in gastric samples. Since EBV is a widespread pathogen and NGS could detect low levels of contaminant virus, sequencing data were confirmed via two PCR tests of increased sensitivity. EBV was detected in the tumour, but not in tumour-adjacent tissue or peripheral mononuclear cells of one GC patient. EBV usually resides in B-cells in frequencies estimated between one-20 cells per million (Rickinson \& Kieff 2007), a frequency of infection that was under the limit of detec- 
tion of either PCR (Ryan et al. 2009, Martínez-López et al. 2014). The enrichment of EBV infection observed in tumour tissue is in agreement with the known direct oncogenic mechanism of EBV through expression of viral oncogenes within the transformed cell. Multiple lines of evidence now support a role for EBV in GC and a recent meta-analysis reports a $10 \%$ world-wide prevalence of EBV associated GCs (Murphy et al. 2009, Camargo et al. 2011). We found a similar frequency of EBV infection in Mexican GC samples (Martínez-López et al. 2014). In EBV associated lymphomas, the number of viral copies has been estimated in 50 viral episomes per tumour cell (Gulley et al. 1994), while seven viral copies were found in an EBV associated nasopharyngeal carcinoma (Liu et al. 2011), which is within the limits of the PCR test used here. It is possible that gastric carcinoma more closely resembles NPC, which highlights the power of the NGS even in pools of tissues from different patients.

Like EBV, HHV6 also belongs to the Herpesviridae family and it is also a highly common infection. HHV6 is the causative agent of roseola infantum and together with HHV7 are classified as the human roseoloviruses. HHV6 has been associated with the nodular sclerosis form of Hodgkin's lymphoma (Siddon et al. 2012), although its role in other tumours is unknown. Our NGS result does not support an oncogenic role for HHV6 in $\mathrm{BrC}$, since viral sequences were found in both $\mathrm{BrC}$ and tumour-adjacent tissues. There are evidences for a modulatory role in tumour development for HHV6; for instance, HHV6-induced secretion of interleukin-2 causes T-cell leukaemias to progress more rapidly (Ojima et al. 2005). Contrary to EBV, HHV6 presents a wide range of tropism, infecting all types of immune cells, neurons, and fibroblasts (Yamanishi et al. 2007). HHV6 increased infection/reactivation often occurs in immunosuppressed individuals. Since we observed HHV6 infection specific to $\mathrm{BrC}$ but no GC patients, cancer induced immunosuppression would not explain the enrichment of HHV6 in breast tissue; although our observation is more in line with HHV6 infecting nontumour cells. HHV6 infection may promote inflammation and thus indirectly participate in tumour growth as it has been recently shown for $\beta$ and $\gamma$ herpesviruses (Abate et al. 2015, Pandya et al. 2015). Still, our data is a preliminary observation that needs to be addressed in future studies. In any case, our work also shows the importance of studying in parallel tumour and tumour-adjacent tissues to be able to identify the specificity of the viral infection and the plausibility of its association with cancer development.

Different studies also support an association of HPV with GC and HPV, EBV, and MMTV with BrC. However, reports have been highly variable, with evidence in favour or against, and the issue remains controversial (Sasco et al. 1993, Wang et al. 1995, Zapata-Benavides et al. 2007, Park et al. 2011). Although, some studies support up to $80 \%$ of $\mathrm{BrC}$ with an infectious aetiology (Joshi et al. 2009) and even infection by multiple viruses (Glenn et al. 2012), we did not find evidence of these associations. According with our results, Tang et al. (2013) analysed transcriptome sequencing reads from $810 \mathrm{BrC}$ tumours finding no support for viral aetiology.
A recent study by Widschwendter et al. (2004) found that secondary $\mathrm{BrC}$ after invasive cervical cancer is importantly associated with the presence of HPV DNA, suggesting viral spread and a possible etiological role for HPV. Widschwendter et al. (2004) highlight the importance of knowing the previous infection history of the patients. We do not know the EBV or HPV infection status of the patients. However, both viruses are highly prevalent worldwide. In Mexico, tissue-specific HPV detection has been performed for research purposes in patients with cervical, anal, oral, and other HPV-related cancers, as well as in individuals with human immunodeficiency virus infection (Berumen et al. 2001, Volkow et al. 2001, Anaya-Saavedra et al. 2008, Méndez-Martínez et al. 2014). In cervical cancer, HPV has been found in close to $100 \%$ of samples supporting the high prevalence of infection in the adult population. Similarly, EBV is a ubiquitous agent infecting approximately $95 \%$ of the adult population worldwide (Henle et al. 1969). In Mexico, there are not population-based studies of EBV seroprevalence, but in our group we analysed Mexican patients with diagnoses of NAG, GC, and premalignant lesions, finding 94.2\% of EBV positive cases (CárdenasMondragón et al. 2015). Therefore, it is possible that in this study all patients were infected with HPV and EBV.

We observed a significant difference in the sensitivity of emulated vs. real sequencing. For example, in the case of EBV screening, the number of viral hits found in silico was of several hundred while only 10 hits were found in the experimental sequencing (equivalent to 50 hits if we consider that the sequenced sample was a pool of 5 genomes). That means than between the number of synthetic and real reads there is roughly a log difference. Thus, virus whose genomes is short and may be present as low copy number as MMTV could have been lost in experimental sequencing. Therefore, although in silico preliminary analyses can help to predict the scope of experimental strategies, we recommend considering this difference in future studies. Notably, we found no reads for MMTV and HPV and we confirmed the absence of MMTV in BrC by PCR (Morales-Sánchez et al. 2013). In this study, we developed and in silico pipeline of analysis of viral sequences in tumour samples, which was then used to sequence $\mathrm{BrC}$ and $\mathrm{GC}$ tumours samples, finding evidence of EBV in $\mathrm{GC}$, a result that validated the sequencing and analysis strategy. An etiological role for EBV in GC is now accepted. Using deep sequencing, The Cancer Genome Atlas (TCGA) research network has identified an EBV+ molecular subgroup of gastric adenocarcinoma in a large set of GC specimens. The $\mathrm{EBV}+\mathrm{GC}$ is tightly clustered by a characteristic viral gene expression program abundant in EBV BART microRNAs expression, $\mathrm{CpG}$ island hypermetilation, PI3K mutations, and PD-L1/2 overexpression (CGARN 2014). Our study supports that EBV also associates with GC in Mexican population, which was not included in TCGA study. NGS technologies together with rigorous analysis by web-based bioinformatics pipelines will become an invaluable tool to identify causative associations in multiple diseases. The design of optimal strategies will help to reduce biological, economical, and computation- 
al resources and, importantly, it may facilitate to translate sequencing technologies into clinical application in countries with limited resources.

\section{REFERENCES}

Abate F, Ambrosio MR, Mundo L, Laginestra MA, Fuligni F, Rossi M, Zairis S, Gazaneo S, de Falco G, Lazzi S, Bellan C, Rocca BJ, Amato T, Marasco E, Etebari M, Ogwang M, Calbi V, Ndede I, Patel K, Chumba D, Piccaluga PP, Pileri S, Leoncini L, Rabadan $\mathrm{R}$ 2015. Distinct viral and mutational spectrum of endemic burkitt lymphoma. PLoS Pathog 11: e1005158.

Agelli M, Clegg LX, Becker JC, Rollison DE 2010. The etiology and epidemiology of merkel cell carcinoma. Curr Probl Cancer 34: 14-37.

Altschul SF, Gish W, Miller W, Myers EW, Lipman DJ 1990. Basic local alignment search tool. J Mol Biol 215: 403-410.

Anaya-Saavedra G, Ramírez-Amador V, Irigoyen-Camacho ME, García-Cuellar CM, Guido-Jiménez M, Méndez-Martínez R, García-Carrancá A 2008. High association of human papillomavirus infection with oral cancer: a case-control study. Arch Med Res 39: 189-197.

Berumen J, Ordoñez RM, Lazcano E, Salmeron J, Galvan SC, Estrada RA, Yunes E, García-Carrancá A, González-Lira G, de la Campa AM 2001. Asian-American variants of human papillomavirus 16 and risk for cervical cancer: a case-control study. J Natl Cancer Inst 93: 1325-1330.

Camargo MC, Murphy G, Koriyama C, Pfeiffer RM, Kim WH, Herrera-Goepfert R, Corvalan AH, Carrascal E, Abdirad A, Anwar M, Hao Z, Kattoor J, Yoshiwara-Wakabayashi E, Eizuru Y, Rabkin CS, Akiba S 2011. Determinants of Epstein-Barr virus-positive gastric cancer: an international pooled analysis. Br J Cancer 105: 38-43.

Camorlinga-Ponce M, Flores-Luna L, Lazcano-Ponce E, Herrero R, Bernal-Sahagun F, Abdo-Francis JM, Aguirre-García J, Muñoz N, Torres J 2008. Age and severity of mucosal lesions influence the performance of serologic markers in Helicobacter pyloriassociated gastroduodenal pathologies. Cancer Epidemiol Biomarkers Prev 17: 2498-2504.

Cárdenas-Mondragón MG, Torres J, Flores-Luna L, CamorlingaPonce M, Carreón-Talavera R, Gomez-Delgado A, Kasamatsu E, Fuentes-Pananá EM 2015. Case-control study of Epstein-Barr virus and Helicobacter pylori serology in Latin American patients with gastric disease. Br J Cancer 112: 1866-1873.

CGARN - Cancer Genome Atlas Research Network 2014. Comprehensive molecular characterization of gastric adenocarcinoma. Nature 513: 202-209.

Chaisson MJ, Pevzner PA 2008. Short read fragment assembly of bacterial genomes. Genome Res 18: 324-330.

Correa P, Piazuelo MB, Camargo MC 2006. Etiopathogenesis of gastric cancer. Scand J Surg 95: 218-224.

Crawford DH 2005. An introduction to viruses and cancer. Microbiology today 5: 110-112.

Crew KD, Neugut AI 2006. Epidemiology of gastric cancer. World $J$ Gastroenterol 12: 354-362.

Feng H, Shuda M, Chang Y, Moore PS 2008. Clonal integration of a polyomavirus in human Merkel cell carcinoma. Science 319: 1096-1100.

Ferlay J, Soerjomataram I, Dikshit R, Eser S, Mathers C, Rebelo M, Parkin DM, Forman D, Bray F 2014. Cancer incidence and mortality worldwide: sources, methods and major patterns in GLOBOCAN 2012. Int J Cancer 136: E359-E386.
Fuentes-Pananá EM, Bannish G, Shah N, Monroe JG 2004. Basal Igalpha/Igbeta signals trigger the coordinated initiation of pre-B cell antigen receptor-dependent processes. J Immunol 173: 1000-1011.

Glenn WK, Heng B, Delprado W, Iacopetta B, Whitaker NJ, Lawson JS 2012. Epstein-Barr virus, human papillomavirus and mouse mammary tumour virus as multiple viruses in breast cancer. PLOS ONE 7: e48788.

Gulley ML, Eagan PA, Quintanilla-Martinez L, Picado AL, Smir BN, Childs C, Dunn CD, Craig FE, Williams Jr JW, Banks PM 1994. Epstein-Barr virus DNA is abundant and monoclonal in the Reed-Sternberg cells of Hodgkin's disease: association with mixed cellularity subtype and Hispanic American ethnicity. Blood 83: 1595-1602.

Henle G, Henle W, Clifford P, Diehl V, Kafuko GW, Kirya BG, Klein G, Morrow RH, Munube GM, Pike P, Tukei PM, Ziegler JL 1969. Antibodies to Epstein-Barr virus in Burkitt's lymphoma and control groups. J Natl Cancer Inst 43: 1147-1157.

Huang W, Li L, Myers JR, Marth GT 2012. ART: a next-generation sequencing read simulator. Bioinformatics 28: 593-594.

Joshi D, Buehring GC 2012. Are viruses associated with human breast cancer? Scrutinizing the molecular evidence. Breast Cancer Res Treat 135: 1-15.

Joshi D, Quadri M, Gangane N, Joshi R, Gangane N 2009. Association of Epstein-Barr virus infection (EBV) with breast cancer in rural Indian women. PLoS ONE 4: e8180.

Kim HH, van den Heuvel AP, Schmidt JW, Ross SR 2011. Novel common integration sites targeted by mouse mammary tumor virus insertion in mammary tumors have oncogenic activity. PLoS ONE 6: e27425.

Kokkola A, Kosunen TU, Puolakkainen P, Sipponen P, Harkonen M, Laxen F, Virtamo J, Haapiainen R, Rautelin H 2003. Spontaneous disappearance of Helicobacter pylori antibodies in patients with advanced atrophic corpus gastritis. APMIS 111: 619-624.

Kostic AD, Ojesina AI, Pedamallu CS, Jung J, Verhaak RG, Getz G, Meyerson M 2011. PathSeq: software to identify or discover microbes by deep sequencing of human tissue. Nat Biotechnol 29: 393-396.

Kubista E 2001. Breast cancer: figures and facts. Wien Med Wochensch 151: 548-551.

Labrecque LG, Barnes DM, Fentiman IS, Griffin BE 1995. EpsteinBarr virus in epithelial cell tumors: a breast cancer study. Cancer Res 55: 39-45.

Langmead B, Trapnell C, Pop M, Salzberg SL 2009. Ultrafast and memory-efficient alignment of short DNA sequences to the human genome. Genome Biol 10: R25.

Liu P, Fang X, Feng Z, Guo YM, Peng RJ, Liu T, Huang Z, Feng Y, Sun X, Xiong Z, Guo X, Pang SS, Wang B, Lv X, Feng FT, Li DJ, Chen LZ, Feng QS, Huang WL, Zeng MS, Bei JX, Zhang Y, Zeng YX 2011. Direct sequencing and characterization of a clinical isolate of Epstein-Barr virus from nasopharyngeal carcinoma tissue by using next-generation sequencing technology. J Virol 85: 11291-11299.

Martínez-López JL, Torres J, Camorlinga-Ponce M, Mantilla A, Leal YA, Fuentes-Pananá EM 2014. Evidence of Epstein-Barr virus association with gastric cancer and non-atrophic gastritis. $\mathrm{Vi}$ ruses 6: 301-318.

Méndez-Martínez R, Rivera-Martínez NE, Crabtree-Ramírez B, Sierra-Madero JG, Caro-Vega Y, Galván SC, de León DC, GarcíaCarrancá A 2014. Multiple human papillomavirus infections are highly prevalent in the anal canal of human immunodeficiency virus-positive men who have sex with men. BMC Infect Dis 14: 671. 
Morales-Sánchez A, Fuentes-Pananá EM 2014. Human viruses and cancer. Viruses 6: 4047-4079.

Morales-Sánchez A, Molina-Muñoz T, Martínez-López JL, HernándezSancen P, Mantilla A, Leal YA, Torres J, Fuentes-Pananá EM 2013. No association between Epstein-Barr virus and mouse mammary tumor virus with breast cancer in Mexican women. Sci Rep 3: 2970.

Morris VL, Medeiros E, Ringold GM, Bishop JM, Varmus HE 1977. Comparison of mouse mammary tumor virus-specific DNA in inbred, wild, and Asian mice, and in tumors and normal organs from inbred mice. J Mol Biol 114: 73-91.

Murphy G, Pfeiffer R, Camargo MC, Rabkin CS 2009. Meta-analysis shows that prevalence of Epstein-Barr virus-positive gastric cancer differs based on sex and anatomic location. Gastroenterology 137: 824-833.

Ojima T, Abe K, Ohyashiki JH, Shirakata M, Yamamoto K 2005. IL2-regulated persistent human herpesvirus-6B infection facilitates growth of adult T-cell leukemia cells. J Med Dent Sci 52: 135-141.

Pandya D, Mariani M, He S, Andreoli M, Spennato M, Dowell-Martino C, Fiedler P, Ferlini C 2015. Epstein-Barr virus microRNA expression increases aggressiveness of solid malignancies. PLoS ONE 10: e 0136058 .

Park DJ, Southey MC, Giles GG, Hopper JL 2011. No evidence of MMTV-like env sequences in specimens from the Australian breast cancer family study. Breast Cancer Res Treat 125: 229-235.

Rickinson AB, Kieff E 2007. Epstein-Barr virus. In DM Knipe, PM Howley (Eds.), Fields virology, 5th ed., Lippincott-Raven Publishers, Philadelphia, p. 2655-2700.

Ryan JL, Morgan DR, Domínguez RL, Thorne LB, Elmore SH, Mino-Kenudson M, Lauwers GY, Booker JK, Gulley ML 2009. High levels of Epstein-Barr virus DNA in latently infected gastric adenocarcinoma. Lab Invest 89: 80-90.

Sasco AJ, Lowenfels AB, Jong PP 1993. Review article: epidemiology of male breast cancer. A meta-analysis of published case-control studies and discussion of selected aetiological factors. Int J Cancer 53: 538-549.
Siddon A, Lozovatsky L, Mohamed A, Hudnall SD 2012. Human herpesvirus 6 positive Reed-Sternberg cells in nodular sclerosis Hodgkin lymphoma. Br J Haematol 158: 635-643.

Tang KW, Alaei-Mahabadi B, Samuelsson T, Lindh M, Larsson E 2013. The landscape of viral expression and host gene fusion and adaptation in human cancer. Nat Commun 4: 2513.

Theelen W, Reijans M, Simons G, Ramaekers FC, Speel EJ, Hopman AH 2010. A new multiparameter assay to assess HPV 16/18, viral load, and physical status together with gain of telomerase genes in HPV-related cancers. Int J Cancer 126: 959-975.

Towner JS, Sealy TK, Khristova ML, Albarino CG, Conlan S, Reeder SA, Quan PL, Lipkin WI, Downing R, Tappero JW, Okware S, Lutwama J, Bakamutumaho B, Kayiwa J, Comer JA, Rollin PE, Ksiazek TG, Nichol ST 2008. Newly discovered ebola virus associated with hemorrhagic fever outbreak in Uganda. PLoS Pathog 4: e1000212.

Volkow P, Rubi S, Lizano M, Carrillo A, Vilar-Compte D, GarcíaCarrancá A, Sotelo R, García B, Sierra-Madero J, Mohar A 2001. High prevalence of oncogenic human papillomavirus in the genital tract of women with human immunodeficiency virus. Gynecol Oncol 82: 27-31.

Wang Y, Holland JF, Bleiweiss IJ, Melana S, Liu X, Pelisson I, Cantarella A, Stellrecht K, Mani S, Pogo BG 1995. Detection of mammary tumor virus env gene-like sequences in human breast cancer. Cancer Res 55: 5173-5179.

Widschwendter A, Brunhuber T, Wiedemair A, Mueller-Holzner E, Marth C 2004. Detection of human papillomavirus DNA in breast cancer of patients with cervical cancer history. J Clin Virol 31: 292-297.

Yamanishi K, Mori Y, Pellet PE 2007. Human herpesvirus 6 and 7. In DM Knipe, PM Howley (eds.), Fields virology, 5th ed., Lippincott-Raven Publishers, Philadelphia, p. 2819-2845.

Zapata-Benavides P, Saavedra-Alonso S, Zamora-Avila D, VargasRodarte C, Barrera-Rodríguez R, Salinas-Silva J, RodríguezPadilla C, Tamez-Guerra R, Trejo-Avila L 2007. Mouse mammary tumor virus-like gene sequences in breast cancer samples of Mexican women. Intervirology 50: 402-407. 